\title{
GUI Based Optimal Drumming Algorithm for Optical Ground Wire (OPGW) Cables
}

\author{
Esmaeil Ebrahimi", Masoud Abdolhosseinpour, Davood Moradi, Faramarz Ghelichi \\ Monenco Iran Consulting Engineers, Tehran, Iran \\ *Corresponding Author: Ebrahimi.Esmaeil@monenco.com
}

Copyright (c) 2013 Horizon Research Publishing All rights reserved.

\begin{abstract}
Optical Ground Wire (OPGW) which has features of both communication channel and lightning-line has been widely used in the high-voltage transmission lines for more than a decade. The optimum use of OPGW cable is necessary to decrease final installation cost. Since OPGW cable wastage is an important key to increase project cost, so in this paper, the genetic algorithm is used to optimize OPGW cables length wastage for drumming purpose. All simulations are presented in user friendly environment as MATLAB/Graphical User Interface (GUI).
\end{abstract}

Keywords Optical Ground Wire, Genetic Algorithm, Graphical User Interface, Drumming

\section{Introduction}

Tavanir a generation and transmission power utility in Iran, which is one of the biggest power companies in Iran, has installed optical ground wire cables (OPGW) in their electrical network. Iran has about $2200 \mathrm{~km}$ of overhead transmission line planned to receive OPGW cables in the next years.

OPGW consists of optical fibers and is covered by steel and aluminium conductor. Optical fiber consists of glass core of about 9 microns in diameter surrounded by a coating called Cladding [1]. It gives a secured communication channel with large traffic capacity, free from electromagnetic interference, induced voltages and atmospheric disturbances which are normally experienced in utility operational environment. OPGW cable is a special kind of electrical ground wire. By combining the function of grounding and data communication into one cable, OPGW deployment considerably reduce the cost of overhead transmission system [2]. This expressway is used for automation of electrical network.

In order to carry out the replacement of the ground wire on transmission lines the following particular requirements will have to be fulfilled [3]:

The new ground wire will have to guarantee the mechanical, electrical and dimensional characteristics of the conventional ground wire that is going to be replaced. The structures will have to support the new operating mechanical loads which will be performed by new cable of OPGW type.

The OPGW cables will have to fulfill with the parameters of short circuit of the transmission lines where they will be installed.

The relation between breaking load of the cable and its weight must be guarantees to fulfill with the required sags for the stringing process without the cable tensions exceed the maximum recommended every day stress by the manufacturer.

In [4] a system using OPGW is constructed to obtain three major functions: monitoring meteorological data, industrial TV monitoring for visual observation of weather and fault section location.

Replacement of OPGW cables demands construction works in overhead transmission lines. The disconnection of these lines has increased the costs and demanding long periods of construction works. In [5] the technique of the "Carrier Method" for replacement of the existent ground wire for OPGW cable with the energized circuits is proposed. Most of the studies carried out to explain OPGW installation and maintenance [6-7] and have attempted to show utilities experience in construction works [8]. However, less effort has been made towards optimization the use of OPGW cable. Hence, it seems necessary to develop an algorithm which enables the determination of the optimum OPGW cables length for drumming.

In this paper the OPGW drumming process involved two aspects: the first is the selection of proper tower e.g. tension tower as dead-end to increase the reliability of the project and the second is the selection of optimal cable length to decrease cable wastage and total cost of project. Both aspects considered carefully by genetic algorithm to determine optimum cable wastage value whiles the reliability of the system is satisfied.

\section{Genetic Algorithm}

Genetic algorithm (GA) is one optimization technique which explores the search space widely to attain optimal solution. It operates with a set of population of chromosome which is represented by string of genes. After a series of 
iterative computation, GA attains optimal solution which is based on Darwinian principle of 'survival of the fittest' [9]. In this method, a fitness function is used to evaluate the quality of each chromosome, based on which it is selected for the next generation. Fitness function of each chromosome is evaluated using minimum error of the regression problem. Reproduction, crossover and mutation are the three basic steps in GA. Generating new set of population of chromosome from the total chromosome set until the mating pool is filled, is called reproduction. Here, ranking method is used for reproduction. In this method, the entire set of chromosome gets a rank based on fitness function and the chromosome with the highest rank is selected and included into the new population. After reproduction, crossover operation is performed in which a pair of parent chromosome is taken to produce a pair of child chromosome and is responsible for global search in GA. Here, uniform crossover is used which uses 0.5 mixing ratio between two parents so that the child chromosome has approximately half of the genes from one parent and another half from the other parent with crossover point randomly chosen. Mutation operation is then performed on string of chromosome to maintain diversity and preventing it from local minima convergence [10].

\section{Proposed Algorithm}

In this paper, GA should calculate cables number, cables length and select proper tower for termination and joint box installation.

The optical fiber link lengths are transmission line route lengths from gantry at one ending station to the gantry in the other ending station. The actual cable lengths to be delivered shall take into account various factors such as sag, service loops, splicing, working lengths and wastage etc. Wastage plays an important role in economic aspects of OPGW projects. Therefore, GA is used to attain not only optimal cable wastage but also to satisfy reliability. Selecting proper tower for termination and joint box installation increases reliability. Tension tower compare with suspension one can tolerate extra mechanical loading, so it is proposed to use tension tower as termination for each delivered cable. For this purpose, each chromosome includes two types for genes as shown in Figure. 1. Continues value for cable length with cables number dimension equal to $\mathrm{m}$ and integer value for tension tower selection with tower number dimension equal to $n-m$ should be used. Values for $m, n$ and tension tower percent can be calculated as follow:

$$
\begin{aligned}
& m=\frac{L}{D C L}+2 \\
& n=(P P-C P) \times \frac{T T N}{100} \\
& C P=\frac{T N}{T T N} \times 100
\end{aligned}
$$

where,

L: Transmission line length,

DCL: Delivery Cable Length,

PP: Predefined tension tower Percent value for all chromosomes,

CP: Calculated tension tower Percent value for each chromosome,

TN: Tension towers Number that are used for joint box installation,

TTN: Total Towers Number that are used for joint box installation.

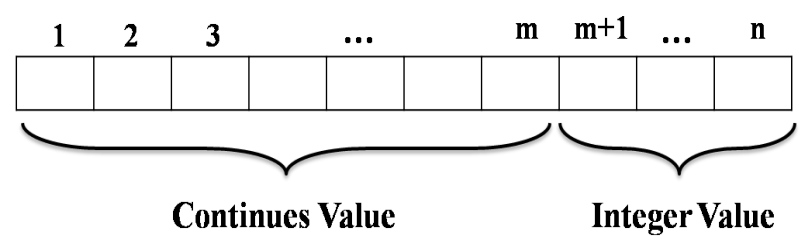

Figure. 1. Chromosome string for GA

It should be noted that two added cable is considered to compensate wastage, but may be not used. In the other hand cable number in final stage is attained by GA.

Generally, the methods used to obtain the correct sag values of the OPGW cables are the same used for conventional ground wire cables. It is assumed that stringing span increased 0.015 percent to compensate sag effects.

The OPGW shall be trained down the tower and to the ground for splicing. The excess length of the OPGW shall not be cut off at this time. To facilitate splicing, the OPGW shall extend a minimum of 10 meters beyond the bottom of the tower.

After completion of sagging and clipping, the surplus OPGW shall be coiled and attached temporarily to the tower. Coils shall be approximately 1 to 1.5 meter in diameter. To avoid degradation of the transmission quality of the optical fibers, the OPGW should not be subjected to excessive pulling tensions or excessively small bend diameters.

Algorithm starts with gantry tower at substation and goes through line route to meet next selected tension tower. If distance between two selected towers is too longer than delivery cable length, algorithm should select suspension tower based on these conditions:

Ruling span ratio for suspension tower must be limited by threshold value.

Adjacent span ratio for suspension tower must be limited by threshold value to avoid longitudinal unbalanced forces.

Ruling span ratio for each suspension tower can be calculated as follows [11]:

$$
R_{S}=\frac{\sqrt{S_{1}^{3}+S_{2}^{3}+\ldots+S_{d}^{3}}}{\sqrt{S_{1}+S_{2}+\ldots+S_{d}}}
$$

where,

S: span length

Rs: ruling span 
d: span number to meet next tension tower or dead end tower.

$$
R_{S-\text { Ratio }}=\frac{R_{S-\text { Right }}}{R_{S-L e f t}}
$$

Longitudinal unbalanced forces can develop at the structures due to various conditions on the line. In rugged terrain, large differentials in adjacent span lengths, combined with inclined spans, could result in significant longitudinal unbalanced load under ice and wind conditions. Non-uniform loading of adjacent spans can also produce longitudinal unbalanced loads. This load is based on an ice shedding condition where ice is dropped from one span and not the adjacent spans.

After selection a tower as dead end tower, algorithm continues to select another tower. This sequence goes on to meet gantry tower in another substation. It should be noted that OPGW cables delivered in a specific range called positive tolerance (Ptolerance) and negative tolerance (Ntolerance).

Each cable's wastage can be calculated as follows:

$$
\begin{aligned}
C W_{i}= & C L_{i}-S L_{i-j} \times 1.015 \\
& -T H_{i}-T H_{j}-20
\end{aligned}
$$

$D C L-N_{\text {tolerance }}<C L_{i}<D C L+P_{\text {tolerance }}$

where,

CW: Cable Wastage,

CL: Cable Length,

DCL: Delivery Cable Length,

SL: Stringing span Length,

TH: Tower Height,

$\mathrm{i}$ and $\mathrm{j}$ : index for selected tower,

After completion of drumming, total cable wastage for each chromosome is attained as follows:

$$
T W=\sum_{k=1}^{m} C W_{k}
$$

where,

TW: Total cables wastage and

$\mathrm{m}$ : cable number.

Selecting some tension towers by GA for installing joint box, forced to cut OPGW for splicing. Increasing splicing number not only degrades the transmission quality of the optical fibers but also imposes additional cost. To avoid this issue, it should be considered weighted fitness function as follows:

$$
F F=\min \left(\begin{array}{c}
W_{1} \times J B N \times C J B \\
+W_{2} \times T W \times C O C
\end{array}\right)
$$

and $W_{1}+W_{2}=1$

where,

FF: Fitness Function,

W1: Joint Box Cost Weight,

W2: Wastage Cost Weight,

JBN: Joint Box Number,
CJB: Cost of one Joint Box installation,

COC: Cost of OPGW Cable per meter.

To meet the predefined tension tower percent, a penalty function is considered as follows:

$$
F F=F F+K \times(|C P-P P|-(C P-P P))
$$

where, $\mathrm{K}$ is a penalty factor and defined based on fitness function value. The flowchart of the proposed algorithm is shown in Figure. 2.

\section{Simulation Results}

As the volume and complexity of data and results continues to grow with the increasing complexity of data sources and algorithms, the need for intuitive representations of that data and results becomes increasingly critical. Graphical User Interface (GUI) is used as user friendly environment to manipulate complex algorithms easily as shown in Figure. 3. In order to test the performance of the proposed algorithm, an actual transmission line data called Anbarabad - Kahnoj in Iran is used. The transmission line is $230 \mathrm{kV}, 60 \mathrm{~km}$.

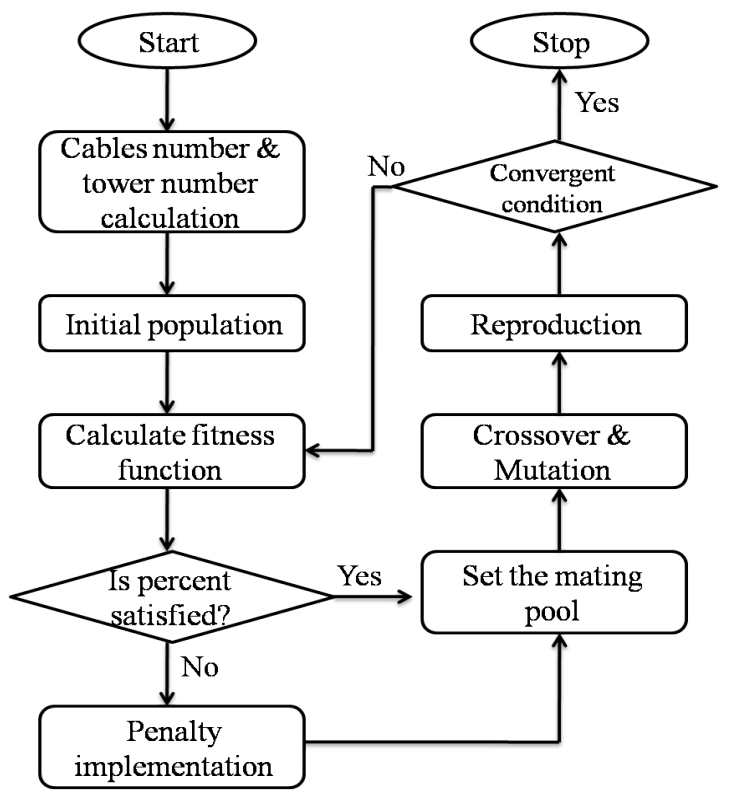

Figure 2. Flowchart of the proposed algorithm

The details of the system parameters are given in Appendix. TABLE 1 shows input data that is used in GUI. All cost units are based on national currency. Based on the input data, $\mathrm{m}$ and $\mathrm{n}$ can calculate as follows:

$$
m=\frac{\text { Line Length }}{\text { Cable Length }}+2=\frac{60 \mathrm{~km}}{5000 \mathrm{~m}}+2=14
$$

To attain value for $\mathrm{n}$, drumming algorithm perform without considering GA. By this method, required towers for splicing will be calculated. In this case, 15 tower need to joint box installation, by considering gantry tower as tension one, the value for $\mathrm{n}$ should be 4 to satisfy predefined percent. 
Table 1. Input data for case study

\begin{tabular}{|c|c|}
\hline Input variable & Value \\
\hline Delivered cable length (m) & 5000 \\
\hline Positive Tolerance (m) & 100 \\
\hline Negative Tolerance (m) & 500 \\
\hline Preset satisfied percent (\%) & 40 \\
\hline Joint Box Cost (Rial) & 12000000 \\
\hline Wastage Cost per meter (Rial) & 50000 \\
\hline W1 & 0.3 \\
\hline W2 & 0.7 \\
\hline Ruling Span Ratio & 1.5 \\
\hline Adjacent Span Ratio & 2 \\
\hline GA Population size & 100 \\
\hline GA Iteration & 2000 \\
\hline
\end{tabular}

GA starts with definition initial population, 14 randomly cable length between $4500 \mathrm{~m}$ and $5100 \mathrm{~m}$ and randomly locate 4 tension tower indexes for each chromosome are used. Algorithm calculates fitness function from (8) and performs penalty function from (9) to compensate fitness value. Crossover and mutation are carried out to produce new offspring. After convergence the GA output data is attained and shown in TABLE 2 . The details of the output parameters are given in Table 3 . It should be noted that wastage for final cable considered as alternative that can be used later.

As shown in TABLE 2, the predefined tension tower percent value is satisfied. In the other side, total cable wastage cost for this case study is 128322350 Rial that is best result in some typical projects in Iran.

As shown in TABLE 3, all selected towers meet the ruling span ratio and the adjacent span ratio, so the result is a high degree of accuracy.

It should be noted that, based on author's knowledge the similar work in this area has not done, so the comparison of the result is not applicable.

Table 2. Output data for case study

\begin{tabular}{|c|c|}
\hline Output variable & Value \\
\hline Used Cable Number & 14 \\
\hline Suspension Tower Number & 9 \\
\hline Tension Tower Number & 6 \\
\hline Section Number & 14 \\
\hline Tension Tower Percent (\%) & 40 \\
\hline Total Cable Wastage (m) & 2566.447 \\
\hline Alternative cable (m) & 2842.719 \\
\hline
\end{tabular}

Table 3. Details of the output parameters for case study

\begin{tabular}{|c|c|c|c|c|c|c|}
\hline $\begin{array}{l}\text { Section } \\
\text { No. }\end{array}$ & First Tower No. & $\begin{array}{c}\text { End Tower } \\
\text { No. }\end{array}$ & $\begin{array}{c}\text { End Tower } \\
\text { Type }\end{array}$ & Calculated Distance (m) & Used Cable (m) & Each Cable wastage (m) \\
\hline 1 & 1 & 10 & $\frac{\mathrm{T}}{\mathrm{T}}$ & 3855.618 & 4525.412 & 6699.794 \\
\hline 2 & 10 & 20 & $\mathrm{~S}$ & 4760.772 & 4813.666 & 52.89408 \\
\hline 3 & 20 & 30 & $\mathrm{~S}$ & 4717.272 & 4778.692 & 61.4195 \\
\hline 4 & 30 & 40 & $\mathrm{~S}$ & 4982.141 & 4983.696 & 1.5554 \\
\hline 5 & 40 & 50 & $\mathrm{~T}$ & 4904.378 & 5017.282 & 112.904 \\
\hline 6 & 50 & 59 & $\mathrm{~S}$ & 4341.454 & 4794.79 & 453.3361 \\
\hline 7 & 59 & 68 & $\mathrm{~T}$ & 4421.152 & 4548.29 & 127.138 \\
\hline 9 & 79 & 89 & $\mathrm{~S}$ & 4690.544 & 5074.565 & 378.7614 \\
\hline 10 & 89 & 99 & $\mathrm{~S}$ & 4755.074 & 4829.381 & 68.28631 \\
\hline 11 & 99 & 109 & $\mathrm{~T}$ & 4828.032 & 5029.732 & 192.8804 \\
\hline 12 & 109 & 119 & $\mathrm{~S}$ & 4716.014 & 4779.804 & 59.63981 \\
\hline 13 & 119 & 128 & $\mathrm{~S}$ & 4402.292 & 4534.369 & 133.4077 \\
\hline 14 & 128 & 132 & $\mathrm{~T}$ & 1906.353 & 4744.582 & 2842.719 \\
\hline
\end{tabular}

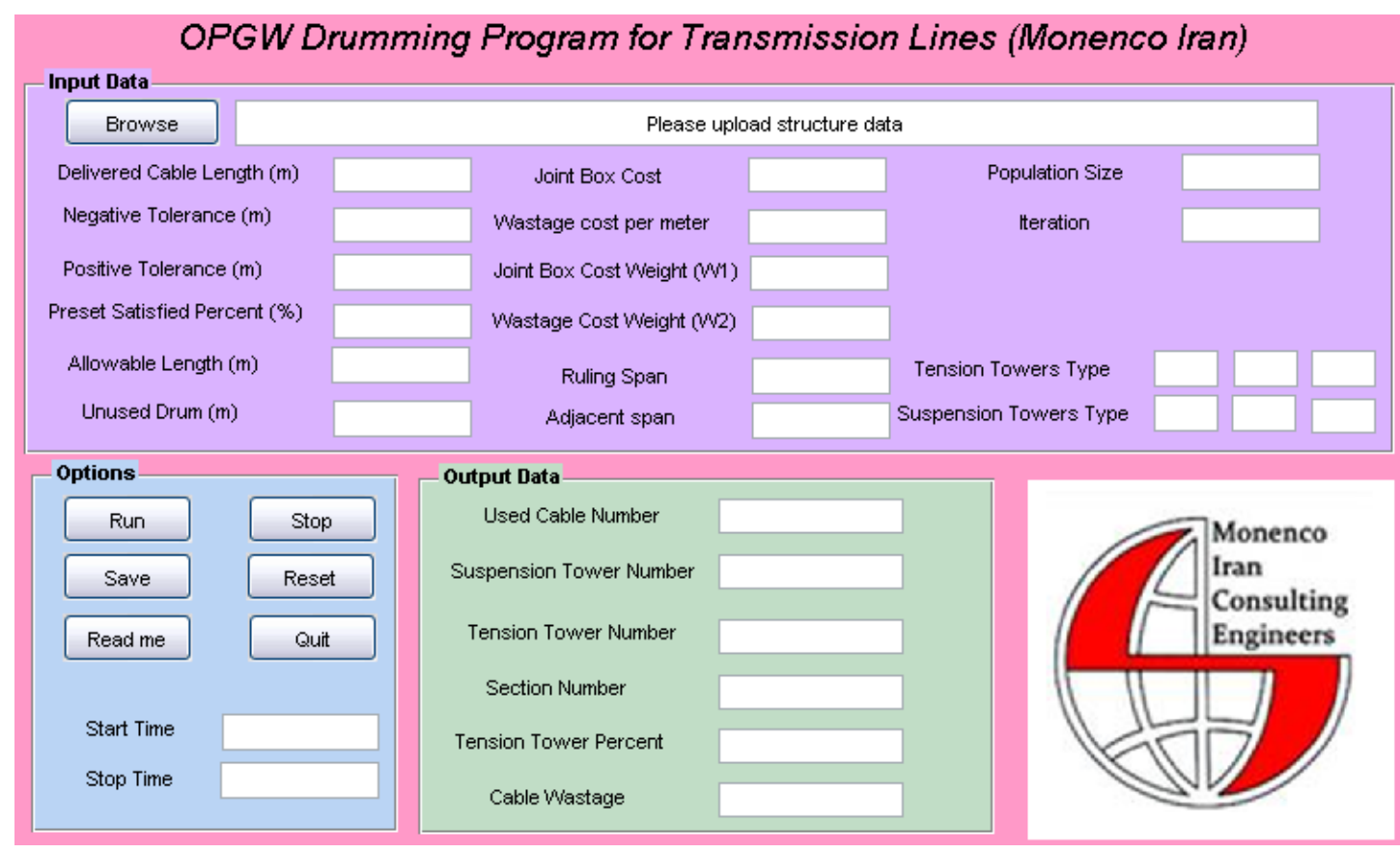

Figure 3. Graphical User Interface (GUI) environment 


\section{Conclusions}

Main idea of this paper was to show that OPGW implementation process is very complex, because some economic problem could arise in exploitation circumstances that are very difficult to understand in phase of solution creation. Choice of cable length as well as splicing should be studied from very beginning of the design process, taking into account financial constraints and taking all necessary measures to eliminate mechanical loading problems. For optimizing the total cost of OPGW implementation, GA is used. Output results show that expected reliability and minimum cable wastage can be attain by GA.

\section{REFERENCES}

[1] IEEE standard for testing and performance of hardware for optical ground wire (OPGW), IEEE Std 1591.1-2012, pp. 1-57, 2012.

[2] C.A. Crisafulli and D. J. Spoor, " A case study on the appropriate selection of optical ground wire " Australasian Universities Power Engineering Conference, AUPEC '08. pp. $1-5,2008$.

[3] S. A. Ali, B. A. Alvi and M. Asif, "OPGW - our experience in KESC" IEEE Electrical Power \& Energy Conference, 2008.

[4] K. Ooura, K. Kanemaru, R. Matsubara and S. Ibuki, " Application of a power line maintenance information system using OPGW to the Nishi-Gunma UHV line" IEEE Trans.
Power Del., Vol. 10, No. 1, Jan. 1995.

[5] F. Nishimura and et.al. "Technology and construction works for OPGW installation in alive-line transmission line" IEEE PES Transmission and Distribution Conference and Exposition, 2003.

[6] S.V. Deshmukh, H.A. Atre, S. Wangde and D. B. Rane, " Fiber optic installation and maintenance " IEEE PES 12th International Conference on Transmission and Distribution Construction, Operation and Live-Line Maintenance (ESMO), pp. 1-5, 2011.

[7] L. J. Fernandez, R. G. Fernandez, M. A. Fernandez Fernandez and C. R. Visser, " Working method for live line optic fiber stringing " IEEE 10th International Conference on Transmission and Distribution Construction, Operation and Live-Line Maintenance, IEEE ESMO. pp. 58-62, 2003.

[8] A. M. Aguiar and M. J. S. Perez, "Experience in OPGW cables selection for overhead transmission live lines" IEEE/PES Transmission \& Distribution Conference and Exposition: Latin America, TDC '06., pp. 1-6, 2006.

[9] J. C. Stacchini de Souza, M. Brown Do Coutto Filho and M. L . Ramos Roberto,"A genetic-based methodology for evaluating requested outages of power network elements" IEEE Trans. Power Sys. Vol. 26, No. 4, pp. 2442 - 2449, 2011.

[10] B. McGinley, J. Maher, C. O'Riordan and F. Morgan, "Maintaining healthy population diversity using adaptive crossover, mutation, and selection" IEEE Trans. Evolutionary Computation, Vol. 15, No. 5, pp. 692 - 714, 2011.

[11] M. Keshavarzian and C. H. Priebe, " Sag and tension calculations for overhead transmission lines at high temperatures - modified ruling span method" IEEE Trans. Power Del., Vol. 15, No. 2, Apr. 2000. 